\title{
Bicriteria Network Design Problems
}

\author{
MadhaV V. Marathe $^{*} \quad$ R. RaVI $^{\dagger} \quad$ RAVi Sundaram $^{\ddagger} \quad$ S. S. RaVi ${ }^{\S}$ \\ DANIEL J. ROSENKRANTZ ${ }^{\S}$ HARRY B. HUNT III $§$
}

August 19, 2019

\begin{abstract}
We study a general class of bicriteria network design problems. A generic problem in this class is as follows: Given an undirected graph and two minimization objectives (under different cost functions), with a budget specified on the first, find a isubgraph from a given subgraph-class that minimizes the second objective subject to the budget on the first. We consider three different criteria - the total edge cost, the diameter and the maximum degree of the network. Here, we present the first polynomial-time approximation algorithms for a large class of bicriteria network design problems for the above mentioned criteria. The following general types of results are presented.

First, we develop a framework for bicriteria problems and their approximations. Second, when the two criteria are the same we present a "black box" parametric search technique. This black box takes in as input an (approximation) algorithm for the unicriterion situation and generates an approximation algorithm for the bicriteria case with only a constant factor loss in the performance guarantee. Third, when the two criteria are the diameter and the total edge costs we use a cluster-based approach to devise a approximation algorithms - the solutions output violate both the criteria by a logarithmic factor. Finally, for the class of treewidth-bounded graphs, we provide pseudopolynomial-time algorithms for a number of bicriteria problems using dynamic programming. We show how these pseudopolynomial-time algorithms can be converted to fully polynomial-time approximation schemes using a scaling technique.
\end{abstract}

AMS 1980 subject classification. 68R10, 68Q15, 68Q25

Keywords. Approximation algorithms, Bicriteria problems, Spanning trees, Network design, Combinatorial algorithms.

${ }^{*}$ Los Alamos National Laboratory, P.O. Box 1663, MS B265, Los Alamos, NM 87545. Email: marat he@ lanl .gov. Research supported by the Department of Energy under Contract W-7405-ENG-36.

${ }^{\dagger}$ GSIA, Carnegie Mellon University, Pittsburgh, PA 15213. Email: ravi+@cmu.edu. Work done at DIMACS, Princeton University, Princeton, NJ 08544. Research supported by a DIMACS postdoctoral fellowship and NSF Career Grant CCR-9625297.

¥ Delta Trading Co. Cambridge, MA. Work done while at LCS, MIT, Cambridge MA 02139. Email: koods@theory. lcs.mit. edu. Research supported by DARPA contract N0014-92-J-1799 and NSF CCR 92-12184.

${ }^{\S}$ Department of Computer Science, University at Albany - SUNY, Albany, NY 12222. Email:\{ravi, djr, hunt \}ecs . albany . edu. Supported by NSF Grants CCR 94-06611 and CCR 90-06396.

I A preliminary version of the the paper appeared in the Proc. 22nd International Colloquium on Automata Languages and Programming, LNCS 944, pp. 487-498 (1995). 


\section{Motivation}

With the information superhighway fast becoming a reality, the problem of designing networks capable of accommodating multimedia (both audio and video) traffic in a multicast (simultaneous transmission of data to multiple destinations) environment has come to assume paramount importance [Ch91, FW+85, KJ83, KP+92A, KP+93]. As discussed in Kompella, Pasquale and Polyzos $[\mathrm{KP}+92 \mathrm{~A}]$, one of the popular solutions to multicast routing involves tree construction. Two optimization criteria - (1) the minimum worst-case transmission delay and (2) the minimum total cost - are typically sought to be minimized in the construction of these trees. Network design problems where even one cost measure must be minimized, are often NP-hard. (See Section A2 on Network Design in GJ79.) But, in real-life applications, it is often the case that the network to be built is required to minimize multiple cost measures simultaneously, with different cost functions for each measure. For example, as pointed out in $[\mathrm{KP}+92 \mathrm{~A}]$, in the problem of finding good multicast trees, each edge has associated with it two edge costs: the construction cost and the delay cost. The construction cost is typically a measure of the amount of buffer space or channel bandwidth used and the delay cost is a combination of the propagation, transmission and queuing delays.

Such multi-criteria network design problems, with separate cost functions for each optimization criterion, also occur naturally in Information Retrieval [BK90] and VLSI designs (see [ZP+94] and the references therein). With the advent of deep micron VLSI designs, the feature size has shrunk to sizes of 0.5 microns and less. As a result, the interconnect resistance, being proportional to the square of the scaling factor, has increased significantly. An increase in interconnect resistance has led to an increase in interconnect delays thus making them a dominant factor in the timing analysis of VLSI circuits. Therefore VLSI circuit designers aim at finding minimum cost (spanning or Steiner) trees given delay bound constraints on source-sink connections.

The above applications set the stage for the formal definition of multicriteria network design problems. We explain this concept by giving a formal definition of a bicriteria network design problem. A generic bicriteria network design problem, $(\mathbf{A}, \mathbf{B}, \mathbf{S})$, is defined by identifying two minimization objectives, - A and B, - from a set of possible objectives, and specifying a membership requirement in a class of subgraphs, - $\mathbf{S}$. The problem specifies a budget value on the first objective, $\mathbf{A}$, under one cost function, and seeks to find a network having minimum possible value for the second objective, $\mathbf{B}$, under another cost function, such that this network is within the budget on the first objective. The solution network must belong to the subgraph-class $\mathbf{S}$. For example, the problem of finding low-cost and low-transmission-delay multimedia networks [KP+92A, KP+93, can be modeled as the (Diameter, Total cost, Spanning tree)-bicriteria problem: given an undirected graph $G=(V, E)$ with two weight functions $c_{e}$ and $d_{e}$ for each edge $e \in E$ modeling construction and delay costs respectively, and a bound $\mathcal{D}$ (on the total delay), find a minimum $c$-cost spanning tree such that the diameter of the tree under the $d$-costs is at most $\mathcal{D}$. It is easy to see that the notion of bicriteria optimization problems can be easily extended to the more general multicriteria optimization problems. In this paper, we will be mainly concerned with bicriteria network design problems. 
In the past, the problem of minimizing two cost measures was often dealt with by attempting to minimize some combination of the two, thus converting it into a unicriterion problem. This approach fails when the two criteria are very disparate. We have chosen, instead, to model bicriteria problems as that of minimizing one criterion subject to a budget on the other. We argue that this approach is both general as well as robust. It is more general because it subsumes the case where one wishes to minimize some functional combination of the two criteria. It is more robust because the quality of approximation is independent of which of the two criteria we impose the budget on. We elaborate on this more in Sections 5.1 and 5.2 .

The organization of the rest of the paper is as follows: Section 3 summarizes the results obtained in this paper; Section 2 discusses related research work; Section 1 contains the hardness results; Section 5.1 shows that the two alternative ways of formulating a given bicriteria problem are indeed equivalent; Section 5.2 demonstrates the generality of the bicriteria approach; Section 6 details the parametric search technique; Section 7 presents the approximation algorithm for diameter constrained Steiner trees; Section 8 contains the results on treewidth-bounded graphs; Section 8 contains some concluding remarks and open problems.

\section{Previous Work}

\subsection{General Graphs}

The area of unicriterion optimization problems for network design is vast and well-explored (See [Ho95, CK95] and the references therein.). Ravi et al. [RM+93] studied the degree-bounded minimum cost spanning tree problem and provided an approximation algorithm with performance guarantee $(O(\log n), O(\log n))$.

The (Degree, Diameter, Spanning tree) problem was studied by Ravi [Ra94] in the context of finding good broadcast networks. There he provides an approximation algorithm for the (Degree, Diameter, Spanning tree) problem with performance guarantee $\left(O\left(\log ^{2} n\right), O(\log n)\right)^{\text {向}}$.

The (Diameter, Total cost, Spanning tree) entry in Table 1 corresponds to the diameter-constrained minimum spanning tree problem introduced earlier. It is known that this problem is NP-hard even in the special case where the two cost functions are identical [HL+89]. Awerbuch, Baratz and Peleg [AB+90] gave an approximation algorithm with $(O(1), O(1))$ performance guarantee for this problem - i.e. the problem of finding a spanning tree that has simultaneously small diameter (i.e., shallow) and small total cost (i.e., light), both under the same cost function. Khuller, Raghavachari and Young [KR+93] studied an extension called Light, approximate Shortest-path Trees (LAST) and gave an approximation algorithm with $(O(1), O(1))$ performance guarantee. Kadaba and Jaffe [KJ83], Kompella et al. [KP+92A], and Zhu et al. [ZP+94] considered the (Diameter, Total cost, Steiner tree) problem with two edge costs and presented heuristics without any guarantees. It is easy to con-

\footnotetext{
${ }^{6}$ The result in Ravi [Ra94] is actually somewhat stronger - given a budget, $D$, on the degree he finds a tree whose total cost is at most $O(\log n)$ times the optimal and whose degree is at most $O\left(D \log n+\log ^{2} n\right)$.
} 
struct examples to show that the solutions produced by these heuristics in [ZP+94, KP+92A], can be arbitrarily bad with respect to an optimal solution. A closely related problem is that of finding a diameter-constrained shortest path between two pre-specified vertices $s$ and $t$, or (Diameter, Total cost, $s$ - $t$ path). This problem, termed the multi-objective shortest path problem (MOSP) in the literature, is NP-complete and Warburton [Wa87] presented the first fully polynomial approximation scheme (FPAS) for it. Hassin [Ha92] provided a strongly polynomial FPAS for the problem which improved the running time of Warburton Wa87]. This result was further improved by Phillips [Ph+93].

The (Total cost, Total cost, Spanning tree)-bicriteria problem has been recently studied by Ganley et al. [GG+95]. They consider a more general problem with more than two weight functions. They also gave approximation algorithms for the restricted case when each weight function obeys triangle inequality. However, their algorithm does not have a bounded performance guarantee with respect to each objective.

\subsection{Treewidth-Bounded Graphs}

Many NP-hard problems have exact solutions when attention is restricted to the class of treewidthbounded graphs and much work has been done in this area (see [AC+93, AL+91, BL+87] and the references therein). Independently, Bern, Lawler and Wong [BL+87] introduced the notion of decomposable graphs. Later, it was shown [AC+93] that the class of decomposable graphs and the class of treewidth-bounded graphs are equivalent. Bicriteria network design problems restricted to treewidth-bounded graphs have been previously studied in [AL+91, Bo88].

\section{Our Contributions}

In this paper, we study the complexity and approximability of a number of bicriteria network design problems. The three objectives we consider are: (i) total cost, (ii) diameter and (iii) degree of the network. These reflect the price of synthesizing the network, the maximum delay between two points in the network and the reliability of the network, respectively. The Total cost objective is the sum of the costs of all the edges in the subgraph. The Diameter objective is the maximum distance between any pair of nodes in the subgraph. The Degree objective denotes the maximum over all nodes in the subgraph, of the degree of the node. The class of subgraphs we consider in this paper are mainly Steiner trees (and hence Spanning trees as a special case); although several of our results extend to more general connected subgraphs such as generalized Steiner trees.

As mentioned in [GJ79], most of the problems considered in this paper, are NP-hard for arbitrary instances even when we wish to find optimum solutions with respect to a single criterion. Given the hardness of finding optimal solutions, we concentrate on devising approximation algorithms with worst case performance guarantees. Recall that an approximation algorithm for a minimization problem $\Pi$ provides a performance guarantee of $\rho$ if for every instance $I$ of $\Pi$, the solution value 
returned by the approximation algorithm is within a factor $\rho$ of the optimal value for $I$. Here, we extend this notion to apply to bicriteria optimization problems. An $(\alpha, \beta)$-approximation algorithm for an $(\mathbf{A}, \mathbf{B}, \mathbf{S})$-bicriteria problem is defined as a polynomial-time algorithm that produces a solution in which the first objective (A) value, is at most $\alpha$ times the budget, and the second objective (B) value, is at most $\beta$ times the minimum for any solution that is within the budget on $\mathbf{A}$. The solution produced must belong to the subgraph-class $\mathbf{S}$. Analogous definitions can be given when $\mathbf{A}$ and/or $\mathbf{B}$ are maximization objectives.

\subsection{General Graphs}

Table 1 contains the performance guarantees of our approximation algorithms for finding spanning trees, S, under different pairs of minimization objectives, $\mathbf{A}$ and $\mathbf{B}$. For each problem cataloged in the table, two different costs are specified on the edges of the undirected graph: the first objective is computed using the first cost function and the second objective, using the second cost function. The rows are indexed by the budgeted objective. For example the entry in row $\mathbf{A}$, column $\mathbf{B}$, denotes the performance guarantee for the problem of minimizing objective $\mathbf{B}$ with a budget on the objective A. All the results in Table 1 extend to finding Steiner trees with at most a constant factor worsening in the performance ratios. For the diagonal entries in the table the extension to Steiner trees follows from Theorem 6.3. ALGORITHM DCST of Section ฤin conjunction with ALGORITHM BICRITERIAEQUiVAlENCE of Section 5.1 yields the (Diameter, Total cost, Steiner tree) and (Total cost, Diameter, Steiner tree) entries. The other nondiagonal entries can also be extended to Steiner trees and these extensions will appear in the journal versions of [RM+93, Ra94]. Our results for arbitrary graphs can be divided into three general categories.

\begin{tabular}{|c|c|c|c|}
\hline Cost Measures & Degree & Diameter & Total Cost \\
\hline Degree & $(O(\log n), O(\log n))^{*}$ & $\left(O\left(\log ^{2} n\right), O(\log n)\right)[\mathrm{Ra} 94]$ & $(O(\log n), O(\log n))[\mathrm{RM}+93]$ \\
\hline Diameter & $\left(O(\log n), O\left(\log ^{2} n\right)\right)[\mathrm{Ra} 94]$ & $\left(1+\gamma, 1+\frac{1}{\gamma}\right)^{*}$ & $(O(\log n), O(\log n))^{*}$ \\
\hline Total Cost & $(O(\log n), O(\log n))[\mathrm{RM}+93]$ & $(O(\log n), O(\log n))^{*}$ & $\left(1+\gamma, 1+\frac{1}{\gamma}\right)^{*}$ \\
\hline
\end{tabular}

Table 1. Performance Guarantees for finding spanning trees in an arbitrary graph on $n$ nodes. Asterisks indicate results obtained in this paper. $\gamma>0$ is a fixed accuracy parameter.

First, as mentioned before, there are two natural alternative ways of formulating general bicriteria problems: (i) where we impose the budget on the first objective and seek to minimize the second and (ii) where we impose the budget on the second objective and seek to minimize the first. We show that an $(\alpha, \beta)$-approximation algorithm for one of these formulations naturally leads to a $(\beta, \alpha)$-approximation algorithm for the other. Thus our definition of a bicriteria approximation is independent of the choice of the criterion that is budgeted in the formulation. This makes it a robust definition and allows us to fill in the entries for the problems $(\mathbf{B}, \mathbf{A}, \mathbf{S})$ by transforming the results for the corresponding problems $(\mathbf{A}, \mathbf{B}, \mathbf{S})$.

Second, the diagonal entries in the table follow as a corollary of a general result (Theorem 6.3) which is proved using a parametric search algorithm. The entry for (Degree, Degree, Spanning tree) 
follows by combining Theorem 6.3 with the $O(\log n)$-approximation algorithm for the degree problem in RM+93]. In [RM+93] they actually provide an $O(\log n)$-approximation algorithm for the weighted degree problem. The weighted degree of a subgraph is defined as the maximum over all nodes of the sum of the costs of the edges incident on the node in the subgraph. Hence we actually get an $(O(\log n), O(\log n))$-approximation algorithm for the (Weighted degree, Weighted degree, Spanning tree)-bicriteria problem. Similarly, the entry for (Diameter, Diameter, Spanning tree) follows by combining Theorem 6.3 with the known exact algorithms for minimum diameter spanning trees [CG82]; while the result for (Total cost, Total cost, Spanning tree) follows by combining Theorem 6.3 with an exact algorithm to compute a minimum spanning tree [CLR].

Finally, we present a cluster based approximation algorithm and a solution based decomposition technique for devising approximation algorithms for problems when the two objectives are different. Our techniques yield $(O(\log n), O(\log n))$-approximation algorithms for the (Diameter, Total cost, Steiner tree) and the (Degree, Total cost, Steiner tree) problems!

\subsection{Treewidth-Bounded Graphs}

We also study the bicriteria problems mentioned above for the class of treewidth-bounded graphs. Examples of treewidth-bounded graphs include trees, series-parallel graphs, $k$-outerplanar graphs, chordal graphs with cliques of size at most $k$, bounded-bandwidth graphs etc. We use a dynamic programming technique to show that for the class of treewidth-bounded graphs, there are either polynomial-time or pseudopolynomial-time algorithms (when the problem is NP-complete) for several of the bicriteria network design problems studied here. A polynomial time approximation scheme (PTAS) for problem $\Pi$ is a family of algorithms $\mathcal{A}$ such that, given an instance $I$ of $\Pi$, for all $\epsilon>0$, there is a polynomial time algorithm $A \in \mathcal{A}$ that returns a solution which is within a factor $(1+\epsilon)$ of the optimal value for $I$. A polynomial time approximation scheme in which the running time grows as a polynomial function of $\epsilon$ is called a fully polynomial time approximation scheme. Here we show how to convert these pseudopolynomial-time algorithms for problems restricted to treewidth-bounded graphs into fully polynomial-time approximation schemes using a general scaling technique. Stated in our notation, we obtain polynomial time approximation algorithms with performance of $(1,1+\epsilon)$, for all $\epsilon>0$. The results for treewidth-bounded graphs are summarized in Table 2. As before, the rows are indexed by the budgeted objective. All algorithmic results in Table 2 also extend to Steiner trees in a straightforward way.

Our results for treewidth-bounded graphs have an interesting application in the context of finding optimum broadcast schemes. Kortsarz and Peleg [KP92 gave $O(\log n)$-approximation algorithms for the minimum broadcast time problem for series-parallel graphs. Combining our results for the (Degree, Diameter, Spanning tree) for treewidth-bounded graphs with the techniques in [Ra94, we obtain an $O\left(\frac{\log n}{\log \log n}\right)$-approximation algorithm for the minimum broadcast time problem for treewidth-bounded graphs (series-parallel graphs have a treewidth of 2), improving and generalizing

\footnotetext{
${ }^{7}$ The result for (Degree, Total cost, Steiner tree) can also be obtained as a corollary of the results in [RM+93].
} 
the result in [KP92]. Note that the best known result for this problem for general graphs is by Ravi [Ra94 who provides an approximation algorithm performance guarantee $\left(O\left(\log ^{2} n\right), O(\log n)\right)$.

\begin{tabular}{|c|c|c|c|}
\hline Cost Measures & Degree & Diameter & Total Cost \\
\hline Degree & polynomial-time & polynomial-time & polynomial-time \\
\hline Diameter & polynomial-time & $\begin{array}{c}\text { (weakly NP-hard) } \\
(1,1+\epsilon)\end{array}$ & $\begin{array}{c}\text { (weakly NP-hard) } \\
(1,1+\epsilon)\end{array}$ \\
\hline Total Cost & polynomial-time & $\begin{array}{c}\text { (weakly NP-hard) } \\
(1,1+\epsilon)\end{array}$ & $\begin{array}{c}\text { (weakly NP-hard) } \\
(1,1+\epsilon)\end{array}$ \\
\hline
\end{tabular}

Table 2. Bicriteria spanning tree results for treewidth-bounded graphs.

\section{Hardness results}

The problem of finding a minimum degree spanning tree is strongly NP-hard [GJ79]. This implies that all spanning tree bicriteria problems, where one of the criteria is degree, are also strongly NP-hard. In contrast, it is well known that the minimum diameter spanning tree problem and the minimum cost spanning tree problems have polynomial time algorithms (see [CLR] and the references therein).

The (Diameter, Total Cost, Spanning tree)-bicriteria problem is strongly NP-hard even in the case where both cost functions are identical [HL+89]. Here we give the details of the reduction to show that (Diameter, Total Cost, Spanning tree) is weakly NP-hard even for series-parallel graphs (i.e. graphs with treewidth at most 2). Similar reductions can be given to show that (Diameter, Diameter, Spanning tree) and (Total cost, Total cost, Spanning tree) are also weakly NP-hard for series-parallel graphs.

We first recall the definition of the PARTITION problem [GJ79]. As an instance of the PARTITION problem we are given a set $T=\left\{t_{1}, t_{2}, \cdots, t_{n}\right\}$ of positive integers and the question is whether there exists a subset $X \subseteq A$ such that $\sum_{t_{i} \in X} t_{i}=\sum_{t_{j} \in T-X} t_{j}=\left(\sum_{t_{j} \in T} t_{j}\right) / 2$.

Theorem 4.1 (Diameter, Total cost, Spanning tree) is NP-hard for series-parallel graphs.

Proof: Reduction from the PARTITION problem. Given an instance $T=\left\{t_{1}, t_{2}, \cdots, t_{n}\right\}$ of the PARTITION problem, we construct a series parallel graph $G$ with $n+1$ vertices, $v_{1}, v_{2}, \cdots v_{n+1}$ and $2 n$ edges. We attach a pair of parallel edges, $e_{i}^{1}$ and $e_{i}^{2}$, between $v_{i}$ and $v_{i+1}, 1 \leq i \leq n$. We now specify the two cost functions $f$ and $g$ on the edges of this graph; $c\left(e_{i}^{1}\right)=t_{i}, c\left(e_{i}^{2}\right)=0, d\left(e_{i}^{1}\right)=$ $0, d\left(e_{i}^{2}\right)=t_{i}, 1 \leq i \leq n$. Let $\sum_{t_{i} \in T} t_{i}=2 H$. Now it is easy to show that $G$ has a spanning tree of $d$-diameter at most $H$ and total $c$-cost at most $H$ if and only if there is a solution to the original instance $T$ of the PARTITION problem. 
We now show that the (Diameter, Total-cost, Steiner tree) problem is hard to approximate within a logarithmic factor. An approximation algorithm provided in Section 7. There is however a gap between the results of Theorems 4.3 and 7.7. Our non-approximability result is obtained by an approximation preserving reduction from the MIN SET COVER. An instance $(T, X)$ of the MIN SET COVER problem consists of a universe $T=\left\{t_{1}, t_{2}, \ldots, t_{k}\right\}$ and a collection of subsets $X=$ $\left\{X_{1}, X_{2}, \ldots, X_{m}\right\}, \quad X_{i} \subseteq T$, each set $X_{i}$ having an associated cost $c_{i}$. The problem is to find a minimum cost collection of the subsets whose union is $T$.

Fact 4.2 Recently [AS97, RS97] have independently shown the following non-approximability result:

It is $N P$-hard to find an approximate solution to the MIN SET COVER problem, with a universe of size $k$, with performance guarantee better than $\Omega(\ln k)$.

Corollary 4.3 There is an approximation preserving reduction from MIN SET COVER problem to the (Diameter, Total Cost, Steiner tree) problem. Thus:

Unless $P=N P$, given an instance of the (Diameter, Total Cost, Steiner tree) problem with $k$ sites, there is no polynomial-time approximation algorithm that outputs a Steiner tree of diameter at most the bound $D$, and cost at most $R$ times that of the minimum cost diameter- $D$ Steiner tree, for $R<\ln k$.

Proof: We give an approximation preserving reduction from the MIN SET COVER problem to the (Diameter, Total Cost, Steiner tree) problem. Given an instance $(T, X)$ of the MIN SET COVER problem where $T=\left\{t_{1}, t_{2}, \ldots, t_{k}\right\}$ and $X=\left\{X_{1}, X_{2}, \ldots, X_{m}\right\}, \quad X_{i} \subseteq T$, where the cost of the set $X_{i}$ is $c_{i}$, we construct an instance $G$ of the (Diameter, Total Cost, Steiner tree) problem as follows. The graph $G$ has a node $t_{i}$ for each element $t_{i}$ of $T$, a node $x_{i}$ for each set $X_{i}$, and an extra "enforcer-node" $n$. For each set $X_{i}$, we attach an edge between nodes $n$ and $x_{i}$ of $c$-cost $c_{i}$, and $d$-cost 1 . For each element $t_{i}$ and set $X_{j}$ such that $t_{i} \in X_{j}$ we attach an edge $\left(t_{i}, x_{j}\right)$ of $c$-cost, 0 , and $d$-cost, 1 . In addition to these edges, we add a path $P$ made of two edges of $c$-cost, 0 , and $d$-cost, 1 , to the enforcer node $n$ (see Figure 1). The path $P$ is added to ensure that all the nodes $t_{i}$ are connected to $n$ using a path of $d$-cost at most 2. All other edges in the graph are assigned infinite $c$ and $d$-costs. The nodes $t_{i}$ along with $n$ and the two nodes of $P$ are specified to be the terminals for the Steiner tree problem instance. We claim that $G$ has a $c$-cost Steiner tree of diameter at most 4 and cost $\mathcal{C}$ if and only if the original instance $(T, X)$ has a solution of cost $\mathcal{C}$.

Note that any Steiner tree of diameter at most 4 must contain a path from $t_{i}$ to $n$, for all $i$, that uses an edge $\left(x_{j}, n\right)$ for some $X_{j}$ such that $t_{i} \in X_{j}$. Hence any Steiner tree of diameter at most 4 provides a feasible solution of equivalent $c$-cost to the original Set cover instance. The proof now follows from Theorem 4.2

\footnotetext{
${ }^{8}$ There is a mild abuse of notation here but it should not lead to any confusion.
} 


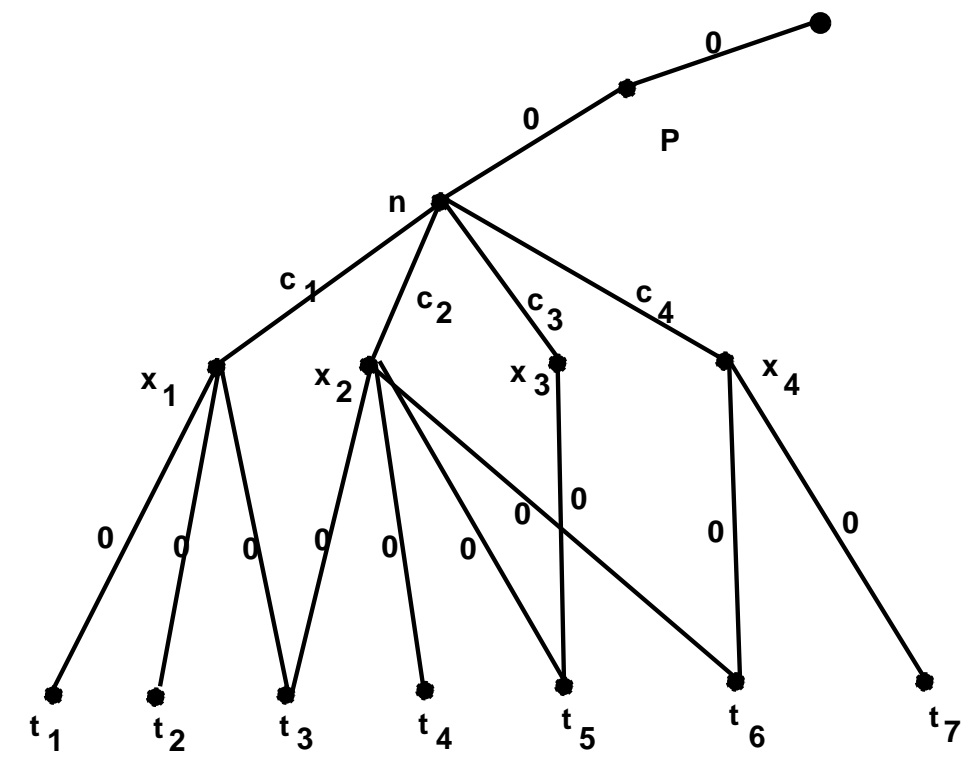

Figure 1: Figure illustrating the reduction from the MIN SET COVER problem to (Diameter, Total cost, Steiner tree) problem. The instance of MIN SET COVER is $(T, X)$ where $T=\left\{t_{1}, t_{2}, \ldots, t_{7}\right\}$, $X=\left\{x_{1}, x_{2}, x_{3}, x_{4}\right\}$. Here $x_{1}=\left\{t_{1}, t_{2}, t_{3}\right\}, x_{2}=\left\{t_{3}, t_{4}, t_{5}\right\}, x_{3}=\left\{t_{5}\right\}$ and $x_{4}=\left\{t_{6}, t_{7}\right\}$. The cost on the edges shown in the figure denotes the $c$-cost of the edges. All these edges have $d$-cost $=1$.

\section{Bicriteria Formulations: Properties}

In Section 1, we claimed that our formulation for bicriteria problems is robust and general. In this section, we justify these claims.

\subsection{Equivalence of Bicriteria Formulations: Robustness}

In this section, we show that our formulation for bicriteria problems is robust and general.

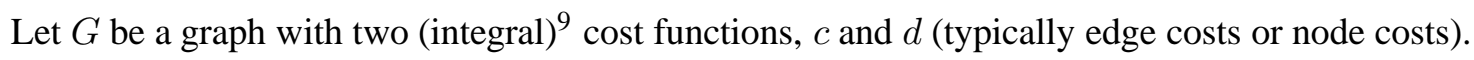
Let $\mathbf{A}(\mathbf{B})$ be a minimization objective computed using cost function $c(d)$. Let the budget bound on the $c$-cost ${ }^{\text {TO }}(d$-cost $)$ of a solution subgraph be denoted by $\mathcal{C}(\mathcal{D})$.

There are two natural ways to formulate a bicriteria problem: (i) $(\mathbf{A}, \mathbf{B}, \mathbf{S})$ - find a subgraph in $\mathbf{S}$ whose $\mathbf{A}$-objective value (under the $c$-cost) is at most $\mathcal{C}$ and which has minimum $\mathbf{B}$-objective value (under the $d$-cost), (ii) ( $\mathbf{B}, \mathbf{A}, \mathbf{S}$ ) - find a subgraph in $\mathbf{S}$ whose $\mathbf{B}$-objective value (under the $d$-cost) is at most $\mathcal{D}$ and which has minimum $\mathbf{A}$-objective value (under the $c$-cost).

Note that bicriteria problems are generally hard, when the two criteria are hostile with respect to each other - the minimization of one criterion conflicts with the minimization of the other. A

\footnotetext{
${ }^{9}$ In case of rational cost functions, our algorithms can be extended with a small additive loss in the performance guarantee.

${ }^{10}$ We use the term "cost under $c$ " or " $c$-cost" in this section to mean the value of the objective function computed using $c$, and not to mean the total of all the $c$ costs in the network.
} 
good example of hostile objectives are the degree and the total edge cost of a spanning tree in an unweighted graph RM+93. Two minimization criteria are formally defined to be hostile whenever the minimum value of one objective is monotonically nondecreasing as the budget (bound) on the value of the other objective is decreased.

Let $\mathbf{A}-\mathbf{A P P R O X}(G, \mathcal{C})$ be any $(\alpha, \beta)$-approximation algorithm for $(\mathbf{A}, \mathbf{B}, \mathbf{S})$ on graph $G$ with budget $\mathcal{C}$ under the $c$-cost. We now show that there is a transformation which produces a $(\beta, \alpha)$ approximation algorithm for $(\mathbf{B}, \mathbf{A}, \mathbf{S})$. The transformation uses binary search on the range of values of the $c$-cost with an application of the given approximation algorithm, A - APPROX, at each step of this search. Let the minimum $c$-cost of a $\mathcal{D}$-bounded subgraph in $\mathbf{S}$ be $O P T_{c}$. Let $\mathcal{C}_{h i}$ be an upper bound on the $c$-cost of any $\mathcal{D}$-bounded subgraph in $\mathbf{S}$. Note that $\mathcal{C}_{h i}$ is at most some polynomial in $n$ times the maximum $c$-cost (of an edge or a node). Hence $\log \left(\mathcal{C}_{h i}\right)$ is at most a polynomial in terms of the input specification. Let $\mathrm{Heu}_{c}\left(\mathrm{Heu}_{d}\right)$ denote the $c$-cost $(d$-cost $)$ of the subgraph output by ALGORITHM BICRITERIA-EQUIVALENCE given below.

\section{ALGORITHM BICRITERIA-EQUIVALENCE:}

- Input: $G$ - graph, $\mathcal{D}$ - budget on criterion B under the $d$-cost, A - APPROX - an $(\alpha, \beta)$ approximation algorithm for $(\mathbf{A}, \mathbf{B}, \mathbf{S})$.

-1. Let $\mathcal{C}_{h i}$ be an upper bound on the $c$-cost of any $\mathcal{D}$-bounded subgraph in $\mathbf{S}$.

2. Do binary search and find a $\mathcal{C}^{\prime}$ in $\left[0, \mathcal{C}_{h i}\right]$ such that

(a) $\mathbf{A}-\operatorname{APPROX}\left(G, \mathcal{C}^{\prime}\right)$ returns a subgraph with $d$-cost greater than $\beta \mathcal{D}$, and

(b) $\mathbf{A}-\operatorname{APPROX}\left(G, \mathcal{C}^{\prime}+1\right)$ returns a subgraph with $d$-cost at most $\beta \mathcal{D}$.

3. If the binary search in Step 2 fails to find a valid $\mathcal{C}^{\prime}$ then output "NO SOLUTION" else output $\mathbf{A}-\operatorname{APPROX}\left(G, \mathcal{C}^{\prime}+1\right)$.

- Output: A subgraph from $\mathbf{S}$ such that its $c$-cost is at most $\alpha$ times that of the minimum $c$-cost $\mathcal{D}$-bounded subgraph and its $d$-cost is at most $\beta \mathcal{D}$.

Claim 5.1 If $G$ contains a $\mathcal{D}$-bounded subgraph in $\mathbf{S}$ then Algorithm BICRITERIA-EQuivalenCE outputs a subgraph from $\mathbf{S}$ whose $c$-cost is at most $\alpha$ times that of the minimum $c$-cost $\mathcal{D}$ bounded subgraph and whose $d$-cost is at most $\beta \mathcal{D}$.

Proof: Since A and B are hostile criteria it follows that the binary search in Step 2 is well defined. Assume that $\mathbf{S}$ contains a $\mathcal{D}$-bounded subgraph. Then, since $\mathbf{A}-\operatorname{APPROX}\left(G, \mathcal{C}_{h i}\right)$ returns a subgraph with $d$-cost at most $\beta \mathcal{D}$, it is clear that ALGORITHM BICRITERIA-EQUIVALENCE outputs a subgraph in this case. As a consequence of Step 2 and the performance guarantee of the approximation algorithm A - APPROX, we get that $\mathcal{C}^{\prime}+1 \leq O P T_{c}$. By Step $2 b$ we have that $H e u_{d} \leq \beta \mathcal{D}$ and $\mathrm{Heu}_{c} \leq \alpha\left(\mathcal{C}^{\prime}+1\right) \leq \alpha O P T_{c}$. Thus ALgORITHM BICRITERIA-EQUiVALENCE outputs a subgraph from $\mathbf{S}$ whose $c$-cost is at most $\alpha$ times that of the minimum $c$-cost $\mathcal{D}$-bounded subgraph and whose $d$-cost is at most $\beta \mathcal{D}$. 
Note however that in general the resulting $(\beta, \alpha)$-approximation algorithm is, not strongly polynomial since it depends on the range of the $c$-costs. But it is a polynomial-time algorithm since its running time is linearly dependent on $\log \mathcal{C}_{h i}$ the largest $c$-cost. The above discussion leads to the following theorem.

Theorem 5.2 Any $(\alpha, \beta)$-approximation algorithm for $(\mathbf{A}, \mathbf{B}, \mathbf{S})$ can be transformed in polynomial time into $\mathbf{a}(\beta, \alpha)$-approximation algorithm for $(\mathbf{B}, \mathbf{A}, \mathbf{S})$.

\subsection{Comparing with other functional combinations: Generality}

Our formulation is more general because it subsumes the case where one wishes to minimize some functional combination of the two criteria. We briefly comment on this next. For the purposes of illustration let $\mathbf{A}$ and $\mathbf{B}$ be two objective functions and let us say that we wish to minimize the sum of the two objectives $\mathbf{A}$ and $\mathbf{B}$. Call this an $(\mathbf{A}+\mathbf{B}, \mathbf{S})$ problem. Let $\mathbf{A}-\mathbf{A P P R O X}(G, \mathcal{C})$ be any $(\alpha, \beta)$-approximation algorithm for $(\mathbf{A}, \mathbf{B}, \mathbf{S})$ on graph $G$ with budget $\mathcal{C}$ under the $c$-cost. We show that $\forall \epsilon>0$, there is a polynomial time $(1+\epsilon) \max \{\alpha, \beta\}$-approximation algorithm for the $(\mathbf{A}+\mathbf{B}, \mathbf{S})$ problem. The transformation uses simple linear search in steps of $(1+\epsilon)$ over the range of values of the $c$-cost with an application of the given approximation algorithm, A - APPROX, at each step of this search. Let the optimum value for the $(\mathbf{A}+\mathbf{B}, \mathbf{S})$ problem on a graph $G$ be $O P T_{c+d}=\left(V_{c}+V_{d}\right)$, where $V_{c}$ and $V_{d}$ denote respectively the contribution of the two costs $c$ and $d$ for $\mathbf{A}$ and $\mathbf{B}$. Let $\mathrm{Heu}_{c}(\mathcal{C})\left(\mathrm{Heu}_{d}(\mathcal{C})\right)$ denote the $c$-cost $(d$-cost) of the subgraph output by $\mathbf{A}-\operatorname{APPROX}(G, \mathcal{C})$. Finally, let $\mathrm{Heu}_{c+d}(\mathcal{C})$ denote the value computed by Algorithm CONVERT.

ALGORITHM CONVERT:

- Input: $G$ - graph, an $\epsilon>0, \mathbf{A}$ - APPROX - an $(\alpha, \beta)$-approximation algorithm for (A, $\mathrm{B}, \mathrm{S})$.

-1. Let $\mathcal{C}_{h i}$ be an upper bound on the $c$-cost of any subgraph in $\mathbf{S}$.

2. Let $R=\left\lceil\log _{(1+\epsilon)} \mathcal{C}_{h i}\right\rceil$

3. For $j=0$ to $R$ do

(a) $M_{j}=(1+\epsilon)^{j}$

(b) Let $\mathrm{Heu}_{c}\left(M_{j}\right), \mathrm{Heu}_{d}\left(M_{j}\right)$ denote the $c$-cost and the $d$-cost of solution obtained by $\operatorname{A}-\operatorname{APPROX}\left(G, M_{j}\right)$.

4. Return the minimum over all $0 \leq j \leq R$, of $\mathcal{F}_{j}=\operatorname{Heu}_{c}\left(M_{j}\right)+\operatorname{Heu}_{d}\left(M_{j}\right)$.

- Output: A subgraph from $\mathbf{S}$ such that the sum of its $c$-cost and its $d$-costs is at most $(1+$ $\epsilon) \max \{\alpha, \beta\}\left(O P T_{c+d}\right)$. 
Theorem 5.3 Let $\mathbf{A}-\operatorname{APPROX}(G, \mathcal{C})$ be any $(\alpha, \beta)$-approximation algorithm for $(\mathbf{A}, \mathbf{B}, \mathbf{S})$ on graph $G$ with budget $\mathcal{C}$ under the $c$-cost. Then, for all $\epsilon>0$, there is a polynomial time $(1+\epsilon) \max \{\alpha, \beta\}$-approximation algorithm for the $(\mathbf{A}+\mathbf{B}, \mathbf{S})$ problem.

Proof Sketch: Consider the iteration of the binary search in which the bound on the $c$-cost is $\mathcal{R}$ such that $V_{c} \leq \mathcal{R} \leq(1+\epsilon) V_{c}$. Notice that such a bound is considered as a result of discretization of the interval $\left[0, \mathcal{C}_{h i}\right]$. Then as a consequence of the performance guarantee of the approximation algorithm A - APPROX, we get that

$$
\operatorname{Heu}_{c}(\mathcal{R}) \leq \alpha \mathcal{R} \leq(1+\epsilon) \alpha V_{c}
$$

By Step 4 , the performance guarantee of the algorithm A - APPROX, and the hostility of $\mathbf{A}$ and $\mathbf{B}$, we have that $H e u_{d}(\mathcal{R}) \leq \beta V_{d}$. Thus $H e u_{c+d}(\mathcal{R}) \leq(1+\epsilon) \alpha V_{c}+\beta V_{d} \leq(1+\epsilon) \max \{\alpha, \beta\}\left(V_{c}+\right.$ $V_{d}$ ). Since Algorithm CONVERT outputs a subgraph from $\mathbf{S}$ the sum of whose $c$-cost and $d$-cost is minimized, we have that

$$
\min _{\mathcal{C}^{\prime} \in\left[0, \mathcal{C}_{h i}\right]}\left(\operatorname{Heu}_{c}\left(\mathcal{C}^{\prime}\right)+\operatorname{Heu}_{d}\left(\mathcal{C}^{\prime}\right)\right) \leq(1+\epsilon) \max \{\alpha, \beta\}\left(O P T_{c+d}\right)
$$

A similar argument shows that an $(\alpha, \beta)$-approximation algorithm $\mathbf{A}-\operatorname{APPROX}(G, \mathcal{C})$, for a $(\mathbf{A}, \mathbf{B}, \mathbf{S})$ problem can be used to find devise a polynomial time $(1+\epsilon)^{2} \alpha \beta$ approximation algorithm for the $(\mathbf{A} \times \mathbf{B}, \mathbf{S})$ problem. A similar argument can also be given for other basic functional combinations. We make two additional remarks.

1. Algorithms for solving $(f(\mathbf{A}, \mathbf{B}), \mathbf{S})$ problems can not in general guarantee any bounded performance ratios for solving the (A, B, S) problem. For example, a solution for the (Total Cost + Total Cost, Spanning Tree) problem or the (Total Cost/Total Cost, Spanning Tree) problem can not be directly used to find a good $(\alpha, \beta)$-approximation algorithm for the (Total Cost, Total Cost, Spanning Tree)-bicriteria problem.

2. The use of approximation algorithms for $(\mathbf{A}, \mathbf{B}, \mathbf{S})$-bicriteria problems, to solve $(f(\mathbf{A}, \mathbf{B}), \mathbf{S})$ problems ( $f$ denotes a function combination of the objectives) does not always yield the best possible solutions. For example problems such as (Total Cost + Total Cost, Spanning Tree) and (Total Cost/Total Cost, Spanning Tree) [Ch77, Me83] can be solved exactly in polynomial time by direct methods but can only be solved approximately using any algorithm for the (Total Cost, Total Cost , Spanning Tree)-bicriteria problem.

\footnotetext{
${ }^{11}$ This is true since the (Total Cost, Total Cost, Spanning Tree)-bicriteria problem is NP-complete and therefore unless $\mathbf{P}=\mathbf{N P}$ cannot be solved in polynomial time.
} 


\section{Parametric Search}

In this section, we present approximation algorithms for a broad class of bicriteria problems where both the objectives in the problem are of the same type (e.g., both are total edge costs of some network computed using two different costs on edges, or both are diameters of some network calculated using two different costs etc.).

As before, let $G$ be a graph with two (integral) cost functions, $c$ and $d$. Let $\mathcal{C}$ denote the budget on criteria A. We assume that the $c$ and $d$ cost functions are of the same kind; i.e., they are both costs on edges or, costs on nodes. Let $\mathbf{U V W}(G, f)$ be any $\rho$-approximation algorithm that on input $G$ produces a solution subgraph in $\mathbf{S}$ minimizing criterion $\mathbf{A}$, under the single cost function $f$. In a mild abuse of notation, we also let $\mathbf{U V W}(G, f)$ denote the $(f$-)cost of the subgraph output by UVW $(G, f)$ when running on input $G$ under cost function $f$. We use the following additional notation in the description of the algorithm and the proof of its performance guarantee. Given constants $a$ and $b$ and two cost functions $f$ and $g$, defined on edges (nodes) of a graph, $a f+b g$ denotes the composite function that assigns a cost $a f(e)+b g(e)$ to each edge (node) in the graph. Let $h(\hat{\mathcal{D}})$ denote the cost of the subgraph, returned by $\mathbf{U V W}\left(G,\left(\frac{\hat{\mathcal{D}}}{\mathcal{C}}\right) c+d\right)$ (under the $\left(\left(\frac{\hat{\mathcal{D}}}{\mathcal{C}}\right) c+d\right)$-cost function). Let the minimum $d$-cost of a $\mathcal{C}$-bounded subgraph in $\mathbf{S}$ be $O P T_{d}$. Let $H e u_{c}\left(H e u_{d}\right)$ denote the $c$-cost ( $d$-cost) of the subgraph output by ALgORITHM PARAMETRIC-SEARCH given below.

Let $\gamma>0$ be a fixed accuracy parameter. In what follows, we devise a $\left((1+\gamma),\left(1+\frac{1}{\gamma}\right)\right)$ approximation algorithm for $(\mathbf{A}, \mathbf{A}, \mathbf{S})$, under the two cost functions $c$ and $d$. The algorithm consists of performing a binary search with an application of the given approximation algorithm, UVW, at each step of this search.

AlgORITHM PARAMETRIC-SEARCH:

- Input: $G$ - graph, $\mathcal{C}$ - budget on criteria $\mathbf{A}$ under the $c$-cost, $\mathbf{U V W}$ - a $\rho$-approximation algorithm that produces a solution subgraph in $\mathbf{S}$ minimizing criterion $\mathbf{A}$, under a single cost function, $\gamma$ - an accuracy parameter.

- 1. Let $\mathcal{D}_{h i}$ be an upper bound on the $d$-cost of any $\mathcal{C}$-bounded subgraph in $\mathbf{S}$.

2. Do binary search and find a $\mathcal{D}^{\prime}$ in $\left[0, \gamma \mathcal{D}_{h i}\right]$ such that

(a) $\mathbf{U V W}\left(G,\left(\frac{\mathcal{D}^{\prime}}{\mathcal{C}}\right) c+d\right)$ returns a subgraph such that $\frac{h\left(\mathcal{D}^{\prime}\right)}{\mathcal{D}^{\prime}}>(1+\gamma) \rho$, and

(b) $\mathbf{U V W}\left(G,\left(\frac{\mathcal{D}^{\prime}+1}{\mathcal{C}}\right) c+d\right)$ returns a subgraph such that $\frac{h\left(\mathcal{D}^{\prime}+1\right)}{\left(\mathcal{D}^{\prime}+1\right)} \leq(1+\gamma) \rho$.

3. If the binary search in Step 2 fails to find a valid $\mathcal{C}^{\prime}$ then output "NO SOLUTION" else output $\mathbf{U V W}\left(G,\left(\frac{\mathcal{D}^{\prime}+1}{\mathcal{C}}\right) c+d\right)$.

- Output: A subgraph from $\mathbf{S}$ such that its $d$-cost is at most $\left(1+\frac{1}{\gamma}\right) \rho$ times that of the minimum $d$-cost $\mathcal{C}$-bounded subgraph and its $c$-cost is at most $(1+\gamma) \rho \mathcal{C}$.

Claim 6.1 The binary search, in Step of ALGORITHM PARAMETRIC-SEARCH is well-defined. 
Proof: $\quad$ Since $\left(\frac{1}{R} \mathbf{U V W}(G, f)\right)$ is the same as $\operatorname{UVW}\left(G, \frac{f}{R}\right)$, we get that $\frac{h(\hat{\mathcal{D}})}{\hat{\mathcal{D}}}=\frac{1}{\hat{\mathcal{D}}} \mathbf{U V W}\left(G,\left(\frac{\hat{\mathcal{D}}}{\mathcal{C}}\right) c+\right.$ $d)=\mathbf{U V W}\left(G,\left(\frac{1}{\mathcal{C}}\right) c+\frac{1}{\hat{\mathcal{D}}} d\right)$. Hence $\frac{h(\hat{\mathcal{D}})}{\hat{\mathcal{D}}}$ is a monotone nonincreasing function of $\hat{D}$. Thus the binary search in Step 2 of ALGORITHM PARAMETRIC-SEARCH is well-defined.

Claim 6.2 If $G$ contains a $\mathcal{C}$-bounded subgraph in $\mathbf{S}$ then AlgORITHM PARAMETRIC-SEARCH outputs a subgraph from $\mathbf{S}$ whose $d$-cost is at most $\left(1+\frac{1}{\gamma}\right) \rho$ times that of the minimum $d$-cost $\mathcal{C}$-bounded subgraph and whose $c$-cost is at most $(1+\gamma) \rho \mathcal{C}$.

Proof: By claim 6.1 we have that the binary search in Step 2 of Algorithm ParametricSEARCH is well-defined.

Assume that $\mathbf{S}$ contains a $\mathcal{C}$-bounded subgraph. Then, since $\mathbf{U V W}\left(G,\left(\frac{\gamma \mathcal{D}_{h i}}{\mathcal{C}}\right) c+d\right)$ returns a subgraph with cost at most $(1+\gamma) \rho \mathcal{D}_{h i}$, under the $\left(\left(\frac{\gamma \mathcal{D}_{h i}}{\mathcal{C}}\right) c+d\right)$-cost function, it is clear that AlgORITHM PARAMETRIC-SEARCH outputs a subgraph in this case.

As a consequence of Step $2 \mathrm{a}$ and the performance guarantee of the approximation algorithm UVW, we get that

$$
\mathcal{D}^{\prime}+1 \leq \frac{O P T_{d}}{\gamma}
$$

By Step 2b we have that the subgraph output by Algorithm PARAMETRIC-SEARCH has the following bounds on the $c$-costs and the $d$-costs.

$$
\begin{gathered}
\text { Heu }_{d} \leq h\left(\mathcal{D}^{\prime}+1\right) \leq \rho(1+\gamma)\left(\mathcal{D}^{\prime}+1\right) \leq\left(1+\frac{1}{\gamma}\right) \rho O P T_{d} \\
\text {Heu}_{c} \leq\left(\frac{\mathcal{C}}{\mathcal{D}^{\prime}+1}\right) h\left(\mathcal{D}^{\prime}+1\right) \leq\left(\frac{\mathcal{C}}{\mathcal{D}^{\prime}+1}\right)(1+\gamma) \rho\left(\mathcal{D}^{\prime}+1\right)=(1+\gamma) \rho \mathcal{C} .
\end{gathered}
$$

Thus Algorithm PARAmetric-Search outputs a subgraph from $\mathbf{S}$ whose $d$-cost is at most $\left(1+\frac{1}{\gamma}\right) \rho$ times that of the minimum $d$-cost $\mathcal{C}$-bounded subgraph and whose $c$-cost is at most $(1+\gamma) \rho \mathcal{C}$.

Note however that the resulting $\left((1+\gamma) \rho,\left(1+\frac{1}{\gamma}\right) \rho\right)$-approximation algorithm for $(\mathbf{A}, \mathbf{A}, \mathbf{S})$ may not be strongly polynomial since it depends on the range of the $d$-costs. But it is a polynomial-time algorithm since its running time is linearly dependent on $\log D_{h i}$. Note that $\mathcal{D}_{h i}$ is at most some polynomial in $n$ times the maximum $d$-cost (of an edge or a node). Hence $\log \left(\mathcal{D}_{h i}\right)$ is at most a polynomial in terms of the input specification.

The above discussion leads to the following theorem.

Theorem 6.3 Any $\rho$-approximation algorithm that produces a solution subgraph in $\mathbf{S}$ minimizing criterion $\mathbf{A}$ can be transformed into a $\left((1+\gamma) \rho,\left(1+\frac{1}{\gamma}\right) \rho\right)$-approximation algorithm for $(\mathbf{A}, \mathbf{A}, \mathbf{S})$.

The above theorem can be generalized from the bicriteria case to the multicriteria case (with appropriate worsening of the performance guarantees) where all the objectives are of the same type but with different cost functions. 


\section{Diameter-Constrained Trees}

In this section, we describe ALGORITHM DCST, our $(O(\log n), O(\log n))$-approximation algorithm for (Diameter, Total cost, Steiner tree) or the diameter-bounded minimum Steiner tree problem. Note that (Diameter, Total cost, Steiner tree) includes (Diameter, Total cost, Spanning tree) as a special case. We first state the problem formally: given an undirected graph $G=(V, E)$, with two cost functions $c$ and $d$ defined on the set of edges, diameter bound $D$ and terminal set $K \subseteq V$, the (Diameter, Total cost, Steiner tree) problem is to find a tree of minimum $c$-cost connecting the set of terminals in $K$ with diameter at most $D$ under the $d$-cost.

The technique underlying ALGORITHM DCST is very general and has wide applicability. Hence, we first give a brief synopsis of it. The basic algorithm works in $(\log n)$ phases (iterations). Initially the solution consists of the empty set. During each phase of the algorithm we execute a subroutine $\Omega$ to choose a subgraph to add to the solution. The subgraph chosen in each iteration is required to possess two desirable properties. First, it must not increase the budget value of the solution by more than $D$; second, the solution cost with respect to $\mathbf{B}$ must be no more than $O P T_{c}$, where $O P T_{c}$ denotes the minimum $c$-cost of a $\mathcal{D}$ bounded subgraph in $\mathbf{S}$. Since the number of iterations of the algorithm is $O(\log n)$ we get a $(\log n, \log n)$-approximation algorithm. The basic technique is fairly straightforward. The non-trivial part is to devise the right subroutine $\Omega$ to be executed in each phase. $\Omega$ must be chosen so as to be able to prove the required performance guarantee of the solution. We use the solution based decomposition technique [Ra94, RM+93] in the analysis of our algorithm. The basic idea (behind the solution based decomposition technique) is to use the existence of an optimal solution to prove that the subroutine $\Omega$ finds the desired subgraph in each phase.

We now present the specifics of ALGORITHM DCST. The algorithm maintains a set of connected subgraphs or clusters each with its own distinguished vertex or center. Initially each terminal is in a cluster by itself. In each phase, clusters are merged in pairs by adding paths between their centers. Since the number of clusters comes down by a factor of 2 each phase, the algorithm terminates in $\left\lceil\log _{2}|K|\right\rceil$ phases with one cluster. It outputs a spanning tree of the final cluster as the solution. 
ALGORITHM DiAMETER-CONSTRAINED-STEINER-TREE (DCST):

- Input: $G=(V, E)$ - graph with two edge cost functions, $c$ and $d, D$ - a bound on the diameter under the $d$-cost, $K \subseteq V$ - set of terminals, $\epsilon$ - an accuracy parameter.

- 1. Initialize the set of clusters $\mathcal{C}_{1}$ to contain $|K|$ singleton sets, one for each terminal in $K$. For each cluster in $\mathcal{C}$, define the single node in the cluster to be the center for the cluster. Initialize the phase count $i:=1$.

2. Repeat until there remains a single cluster in $\mathcal{C}_{i}$

(a) Let the set of clusters $\mathcal{C}_{i}=\left\{C_{1} \ldots, C_{k_{i}}\right\}$ at the beginning of the $i$ 'th phase (observe that $\left.k_{1}=|K|\right)$.

(b) Construct a complete graph $G_{i}$ as follows: The node set $V_{i}$ of $G_{i}$ is $\{v$ : $v$ is the center of a cluster in $\mathcal{C}\}$. Let path $P_{x y}$ be a $(1+\epsilon)$-approximation to the minimum $c$-cost diameter $D$-bounded path between centers $v_{x}$ and $v_{y}$ in $G$. Between every pair of nodes $v_{x}$ and $v_{y}$ in $V_{i}$, include an edge $\left(v_{x}, v_{y}\right)$ in $G_{i}$ of weight equal to the $c$-cost of $P_{x y}$.

(c) Find a minimum-weight matching of largest cardinality in $G_{i}$.

(d) For each edge $e=\left(v_{x}, v_{y}\right)$ in the matching, merge clusters $C_{x}$ and $C_{y}$, for which $v_{x}$ and $v_{y}$ were centers respectively, by adding path $P_{x y}$ to form a new cluster $C_{x y}$. The node (edge) set of the cluster $C_{x y}$ is defined to be the union of the node (edge) sets of $C_{x}, C_{y}$ and the nodes (edges) in $P_{x y}$. One of $v_{x}$ and $v_{y}$ is (arbitrarily) chosen to be the center $v_{x y}$ of cluster $C_{x y}$ and $C_{x y}$ is added to the cluster set $\mathcal{C}_{i+1}$ for the next phase.

(e) $i:=i+1$.

3. Let $C^{\prime}$, with center $v^{\prime}$ be the single cluster left after Step 2 . Output a shortest path tree of $C^{\prime}$ rooted at $v^{\prime}$ using the $d$-cost.

- Output: A Steiner tree connecting the set of terminals in $K$ with diameter at most $2\left\lceil\log _{2} n\right\rceil D$ under the $d$-cost and of total $c$-cost at most $(1+\epsilon)\left\lceil\log _{2} n\right\rceil$ times that of the minimum $c$-cost diameter $D$-bounded Steiner tree.

We make a few points about ALgORITHM DCST:

1. The clusters formed in Step 2d need not be disjoint.

2. All steps, except Step 2b, in algorithm DCST can be easily seen to have running times independent of the weights. We employ Hassin's strongly polynomial FPAS for Step 2b $\mathrm{Ha92}$. Hassin's approximation algorithm for the $D$-bounded minimum $c$-cost path runs in time $O\left(|E|\left(\frac{n^{2}}{\epsilon} \log \frac{n}{\epsilon}\right)\right)$. Thus ALGORITHM DCST is a strongly polynomial time algorithm.

3. Instead of finding an exact minimum cost matching in Step 20, we could find an approximate minimum cost matching [GW95]. This would reduce the running time of the algorithm at the cost of introducing a factor of 2 to the performance guarantee.

We now state some observations that lead to a proof of the performance guarantee of ALGORITHM DCST. Assume, in what follows, that $G$ contains a diameter $D$-bounded Steiner tree. We also refer to each iteration of Step 2 as a phase. 
Claim 7.1 Algorithm DCST terminates in $\left\lceil\log _{2}|K|\right\rceil$ phases.

Proof: Let $k_{i}$ denote the number of clusters in phase $i$. Note that $k_{i+1}=\left\lceil\frac{k_{i}}{2}\right\rceil$ since we pair up the clusters (using a matching in Step $2 \mathrm{~d}$ ). Hence we are left with one cluster after phase $\left\lceil\log _{2}|K|\right\rceil$ and algorithm DCST terminates.

The next claim points out as clusters get merged, the nodes within each cluster are not too far away (with respect to $d$-distance) from the center of the cluster. This intuitively holds for the following important reasons. First, during each phase, the graph $G_{i}$ has as its vertices, the centers of the clusters in that iteration. As a result, we merge the clusters by joining their centers in Step 2d. Second, in Step 2d, for each pair of clusters $C_{x}$ and $C_{y}$ that are merged, we select one of their centers, $v_{x}$ or $v_{y}$ as the center $v_{x y}$ for the merged cluster $C_{x y}$. This allows us to inductively maintain two properties: (i) the required distance of the nodes in a cluster to their centers in an iteration $i$ is $i D$ and (ii) the center of a cluster at any given iteration is a terminal node.

Claim 7.2 Let $C \in \mathcal{C}_{i}$ be any cluster in phase $i$ of algorithm DCST. Let $v$ be the center of $C$. Then any node $u$ in $C$ is reachable from $v$ by a diameter- $i D$ path in $C$ under the $d$-cost.

Proof: Note that the existence of a diameter $D$-bounded Steiner tree implies that all paths added in Step $2 \mathrm{~d}$ have diameter at most $D$ under $d$-cost. The proof now follows in a straightforward fashion by induction on $i$.

Lemma 7.3 Algorithm DCST outputs a Steiner tree with diameter at most $2\left\lceil\log _{2}|K|\right\rceil \cdot D$ under the $d$-cost.

Proof: The proof follows from Claims 7.1 and 7.2 .

This completes the proof of performance guarantee with respect to the $d$-cost. We now proceed to prove the performance guarantee with respect to the $c$-costs. We first recall the following pairing lemma.

Claim 7.4 [RM+93] Let $T$ be an edge-weighted tree with an even number of marked nodes. Then there is a pairing $\left(v_{1}, w_{1}\right), \ldots,\left(v_{k}, w_{k}\right)$ of the marked nodes such that the $v_{i}-w_{i}$ paths in $T$ are edge-disjoint.

Claim 7.5 Let $O P T$ be any minimum $c$-cost diameter- $D$ bounded Steiner tree and let $O P T_{c}$ denote its $c$-cost. The weight of the largest cardinality minimum-weight matching found in Step 20 in each phase $i$ of algorithm DCST is at most $(1+\epsilon) \cdot O P T_{c}$. 
Proof: Consider phase $i$ of algorithm DCST. Note that since the centers at stage $i$ are a subset of the nodes in the first iteration, the centers $v_{i}$ are terminal nodes. Thus they belong to $O P T$. Mark those vertices in $O P T$ that correspond to the matched vertices, $v_{1}, v_{2}, \ldots, v_{2\left\lfloor\frac{k_{i}}{2}\right\rfloor}$ of $G_{i}$ in Step $2 \mathrm{c}$. Then by Claim 7.4 there exists a pairing of the marked vertices, say $\left(v_{1}, v_{2}\right), \ldots,\left(v_{2\left\lfloor\frac{k_{i}}{2}\right\rfloor-1}, v_{2\left\lfloor\frac{k_{i}}{2}\right\rfloor}\right)$, and a set of edge-disjoint paths in OPT between these pairs. Since these paths are edge-disjoint their total $c$-cost is at most $O P T_{c}$. Further these paths have diameter at most $D$ under the $d$-cost. Hence the sum of the weights of the edges $\left(v_{1}, v_{2}\right), \ldots,\left(v_{2\left\lfloor\frac{k_{i}}{2}\right\rfloor-1}, v_{2\left\lfloor\frac{k_{i}}{2}\right\rfloor}\right)$ in $G_{i}$, which forms a perfect matching on the set of matched vertices, is at most $(1+\epsilon) \cdot O P T_{c}$. But in Step 20 of Algorithm DCST, a minimum weight perfect matching in the graph $G_{i}$ was found. Hence the weight of the matching found in Step 2d in phase $i$ of Algorithm DCST is at most $(1+\epsilon) \cdot O P T_{c}$.

Lemma 7.6 Let $O P T$ be any minimum $c$-cost diameter- $D$ bounded Steiner tree and let $O P T_{c}$ denote its $c$-cost. AlgORITHM DCST outputs a Steiner tree with total $c$-cost at most $(1+$ $\epsilon)\left\lceil\log _{2}|K|\right\rceil \cdot O P T_{c}$.

Proof: From Claim 7.5 we have that the $c$-cost of the set of paths added in Step $2 \mathrm{~d}$ of any phase is at most $(1+\epsilon) \cdot O P T_{c}$. By Claim 7.1 there are a total of $\left\lceil\log _{2}|K|\right\rceil$ phases and hence the Steiner tree output by ALGORITHM DCST has total $c$-cost at most $(1+\epsilon)\left\lceil\log _{2}|K|\right\rceil \cdot O P T_{c}$.

From Lemmas 7.3 and 7.6 we have the following theorem.

Theorem 7.7 There is a strongly polynomial-time algorithm that, given an undirected graph $G=(V, E)$, with two cost functions $c$ and $d$ defined on the set of edges, diameter bound $D$, terminal set $K \subseteq V$ and a fixed $\epsilon>0$, constructs a Steiner tree of $G$ of diameter at most $2\left\lceil\log _{2}|K|\right\rceil D$ under the $d$-costs and of total $c$-cost at most $(1+\epsilon)\left\lceil\log _{2}|K|\right\rceil$ times that of the minimum- $c$-cost of any Steiner tree with diameter at most $D$ under $d$.

\section{Treewidth-Bounded Graphs}

In this section we consider the class of treewidth-bounded graphs and give algorithms with improved time bounds and performance guarantees for several bicriteria problems mentioned earlier. We do this in two steps. First we develop pseudopolynomial-time algorithms based on dynamic programming. We then present a general method for deriving fully polynomial-time approximation schemes (FPAS) from the pseudopolynomial-time algorithms. We also demonstrate an application of the above results to the minimum broadcast time problem.

A class of treewidth-bounded graphs can be specified using a finite number of primitive graphs and a finite collection of binary composition rules. We use this characterization for proving our results. A class of treewidth-bounded graphs $\Gamma$ is inductively defined as follows [BL+87]. 
1. The number of primitive graphs in $\Gamma$ is finite.

2. Each graph in $\Gamma$ has an ordered set of special nodes called terminals. The number of terminals in each graph is bounded by a constant, say $k$.

3. There is a finite collection of binary composition rules that operate only at terminals, either by identifying two terminals or adding an edge between terminals. A composition rule also determines the terminals of the resulting graph, which must be a subset of the terminals of the two graphs being composed.

\subsection{Exact Algorithms}

Theorem 8.1 Every problem in Table 2 can be solved exactly in $O\left((n \cdot \mathcal{C})^{O(1)}\right)$-time for any class of treewidth bounded graphs with no more than $k$ terminals, for fixed $k$ and a budget $\mathcal{C}$ on the first objective.

The above theorem states that there exist pseudopolynomial-time algorithms for all the bicriteria problems from Table 2 when restricted to the class of treewidth-bounded graphs. The basic idea is to employ a dynamic programming strategy. In fact, this dynamic programming strategy (in conjunction with Theorem 5.2) yields polynomial-time (not just pseudopolynomial-time) algorithms whenever one of the criteria is the degree. We illustrate this strategy by presenting in some detail the algorithm for the diameter-bounded minimum cost spanning tree problem.

Theorem 8.2 For any class of treewidth-bounded graphs with no more than $k$ terminals, there is an $O\left(n \cdot k^{2 k+4} \cdot \mathcal{D}^{O\left(k^{4}\right)}\right)$-time algorithm for solving the diameter $\mathcal{D}$-bounded minimum $c$-cost spanning tree problem.

Proof: Let $d$ be the cost function on the edges for the first objective (diameter) and $c$, the cost function for the second objective (total cost). Let $\Gamma$ be any class of decomposable graphs. Let the maximum number of terminals associated with any graph $G$ in $\Gamma$ be $k$. Following [BL+87], it is assumed that a given graph $G$ is accompanied by a parse tree specifying how $G$ is constructed using the rules and that the size of the parse tree is linear in the number of nodes.

Let $\pi$ be a partition of the terminals of $G$. For every terminal $i$ let $d_{i}$ be a number in $\{1,2, \ldots, \mathcal{D}\}$. For every pair of terminals $i$ and $j$ in the same block of the partition $\pi$ let $d_{i j}$ be a number in $\{1,2, \ldots, \mathcal{D}\}$. Corresponding to every partition $\pi$, set $\left\{d_{i}\right\}$ and set $\left\{d_{i j}\right\}$ we associate a cost for $G$ defined as follows:

\footnotetext{
$\operatorname{Cost}_{\left\{d_{i}\right\},\left\{d_{i j}\right\}}^{\pi}=$ Minimum total cost under the $c$ function of any forest containing a tree for each block of $\pi$, such that the terminal nodes occurring in each tree are exactly the members of the corresponding block of $\pi$, no pair of trees is connected, every vertex in $G$
} 
appears in exactly one tree, $d_{i}$ is an upper bound on the maximum distance (under the $d$-function) from $i$ to any vertex in the same tree and $d_{i j}$ is an upper bound the distance (under the $d$-function) between terminals $i$ and $j$ in their tree.

For the above defined cost, if there is no forest satisfying the required conditions the value of Cost is defined to be $\infty$.

Note that the number of cost values associated with any graph in $\Gamma$ is $O\left(k^{k} \cdot \mathcal{D}^{O\left(k^{2}\right)}\right)$. We now show how the cost values can be computed in a bottom-up manner given the parse tree for $G$. To begin with, since $\Gamma$ is fixed, the number of primitive graphs is finite. For a primitive graph, each cost value can be computed in constant time, since the number of forests to be examined is fixed. Now consider computing the cost values for a graph $G$ constructed from subgraphs $G_{1}$ and $G_{2}$, where the cost values for $G_{1}$ and $G_{2}$ have already been computed. Notice that any forest realizing a particular cost value for $G$ decomposes into two forests, one for $G_{1}$ and one for $G_{2}$ with some cost values. Since we have maintained the best cost values for all possibilities for $G_{1}$ and $G_{2}$, we can reconstruct for each partition of the terminals of $G$ the forest that has minimum cost value among all the forests for this partition obeying the diameter constraints. We can do this in time independent of the sizes of $G_{1}$ and $G_{2}$ because they interact only at the terminals to form $G$, and we have maintained all relevant information.

Hence we can generate all possible cost values for $G$ by considering combinations of all relevant pairs of cost values for $G_{1}$ and $G_{2}$. This takes time $O\left(k^{4}\right)$ per combination for a total time of $O\left(k^{2 k+4} \cdot \mathcal{D}^{O\left(k^{4}\right)}\right)$. As in [BL+87], we assume that the size of the given parse tree for $G$ is $O(n)$. Thus the dynamic programming algorithm takes time $O\left(n \cdot k^{2 k+4} \cdot \mathcal{D}^{O\left(k^{4}\right)}\right)$. This completes the proof.

\subsection{Fully Polynomial-Time Approximation Schemes}

The pseudopolynomial-time algorithms described in the previous section can be used to design fully polynomial-time approximation schemes (FPAS) for these same problems for the class of treewidthbounded graphs. We illustrate our ideas once again by devising an FPAS for the (Diameter, Total cost, Spanning tree)-bicriteria problem for the class of treewidth-bounded graphs. The basic technique underlying our algorithm, ALGORITHM FPAS-DCST, is approximate binary search using rounding and scaling - a method similar to that used by Hassin [Ha92] and Warburton [Wa87].

As in the previous subsection, let $G$ be a treewidth-bounded graph with two (integral) edgecost functions $c$ and $d$. Let $D$ be a bound on the diameter under the $d$-cost. Let $\epsilon$ be an accuracy parameter. Without loss of generality we assume that $\frac{1}{\epsilon}$ is an integer. We also assume that there exists a $D$-bounded spanning tree in $G$. Let $O P T$ be any minimum $c$-cost diameter $D$-bounded spanning tree and let $O P T_{c}$ denote its $c$-cost. Let $\mathbf{T C S T o n T W}(G, c, d, C)$ be a pseudopolynomial time algorithm for the (Total cost, Diameter, Spanning tree) problem on treewidth-bounded graphs; i.e., 
TCSTonTW outputs a minimum diameter spanning tree of $G$ with total cost at most $C$ (under the $c$-costs). Let the running time of TCSTonTW be $p(n, C)$ for some polynomial $p$. For carrying out our approximate binary search we need a testing procedure PROCEDURE TEST(V) which we detail below:

$\operatorname{PROCEDURE} \operatorname{TeSt}(\lambda)$ :

- Input: $G$ - treewidth bounded graph, $D$ - bound on the diameter under the $d$-cost, $\lambda$ testing parameter, TCSTonTW - a pseudopolynomial time algorithm for the (Total cost, Diameter, Spanning tree) problem on treewidth-bounded graphs, $\epsilon$ - an accuracy parameter.

- 1. Let $\left\lfloor\frac{c}{\lambda \epsilon /(n-1)}\right\rfloor$ denote the cost function obtained by setting the cost of edge $e$ to $\left\lfloor\frac{c_{e}}{\lambda \epsilon /(n-1)}\right\rfloor$.

2. If there exists a $C$ in $\left[0, \frac{n-1}{\epsilon}\right]$ such that $\operatorname{TCSTonTW}\left(G,\left\lfloor\frac{c}{\lambda \epsilon /(n-1)}\right\rfloor, d, C\right)$ produces a spanning tree with diameter at most $D$ under the $d$-cost then output LOW otherwise output HIGH.

- Output: HIGH/LOW.

We now prove that Procedure $\operatorname{Test}(\lambda)$ has the properties we need to do a binary search.

Claim 8.3 If $O P T_{c} \leq \lambda$ then Procedure Test $(\lambda)$ outputs LOW. And, if $O P T_{c}>\lambda(1+\epsilon)$ then Procedure Test $(\lambda)$ outputs HIGH.

Proof: If $O P T_{c} \leq \lambda$ then since

$$
\sum_{e \in O P T}\left\lfloor\frac{c_{e}}{\lambda \epsilon /(n-1)}\right\rfloor \leq \sum_{e \in O P T} \frac{c_{e}}{\lambda \epsilon /(n-1)} \leq \frac{O P T_{c}}{\lambda \epsilon /(n-1)} \leq \frac{n-1}{\epsilon}
$$

therefore Procedure Test $(\lambda)$ outputs LOW.

Let $T_{c}$ be the $c$-cost of any diameter $D$ bounded spanning tree. Then we have $T_{c} \geq O P T_{c}$. If $O P T_{c}>\lambda(1+\epsilon)$ then since

$\sum_{e \in T}\left\lfloor\frac{c_{e}}{\lambda \epsilon /(n-1)}\right\rfloor \geq \sum_{e \in T}\left(\frac{c_{e}}{\lambda \epsilon /(n-1)}-1\right) \geq \frac{T_{c}}{\lambda \epsilon /(n-1)}-(n-1) \geq \frac{O P T_{c}}{\lambda \epsilon /(n-1)}-(n-1)>\frac{n-1}{\epsilon}$

therefore Procedure TEST $(\lambda)$ outputs HIGH.

Claim 8.4 The running time of $\operatorname{Procedure} \operatorname{Test}(\lambda)$ is $O\left(\frac{n}{\epsilon} p\left(n, \frac{n}{\epsilon}\right)\right)$.

Proof: Procedure Test $(\lambda)$ invokes TCSTonTW only $\frac{n-1}{\epsilon}$ times. And each time the budget $C$ is bounded by $\frac{n-1}{\epsilon}$, hence the running time of Procedure Test $(\lambda)$ is $O\left(\frac{n}{\epsilon} p\left(n, \frac{n}{\epsilon}\right)\right)$.

We are ready to describe Algorithm FPAS-DCST - which uses Procedure Test $(\lambda)$ to do an approximate binary search. 
ALGORITHM FPAS-DCST:

- Input: $G$ - treewidth-bounded graph, $D$ - bound on the diameter under the $d$-cost, TCSTonTW - a pseudopolynomial time algorithm for the (Total cost, Diameter, Spanning tree) problem on treewidth-bounded graphs, $\epsilon$ - an accuracy parameter.

- 1. Let $C_{h i}$ be an upper bound on the $c$-cost of any $D$-bounded spanning tree. Let $L B=0$ and $U B=C_{h i}$.

2. While $U B \geq 2 L B$ do

(a) Let $\lambda=(L B+U B) / 2$.

(b) If Procedure Test $(\lambda)$ returns HIGH then set $L B=\lambda$ else set $U B=\lambda(1+\epsilon)$.

3. Run TCSTonTW $\left(G,\left\lfloor\frac{c}{L B \epsilon /(n-1)}\right\rfloor, d, C\right)$ for all $C$ in $\left[0,2\left(\frac{n-1}{\epsilon}\right)\right]$ and among all the trees with diameter at most $D$ under the $d$-cost output the tree with the lowest $c$-cost.

- Output: A spanning tree with diameter at most $D$ under the $d$-cost and with $c$-cost at most $(1+\epsilon)$ times that of the minimum $c$-cost $D$-bounded spanning tree.

Lemma 8.5 If $G$ contains a $D$-bounded spanning tree then ALGORITHM FPAS-DCST outputs a spanning tree with diameter at most $D$ under the $d$-cost and with $c$-cost at most $(1+$ є) $O P T_{c}$.

Proof: It follows easily from Claim 8.3 that the loop in Step 2 of ALGoRITHM FPAS-DCST executes $O\left(\log C_{h i}\right)$ times before exiting with $L B \leq O P T_{c} \leq U B<2 L B$.

Since

$$
\sum_{e \in O P T}\left\lfloor\frac{c_{e}}{L B \epsilon /(n-1)}\right\rfloor \leq \sum_{e \in O P T} \frac{c_{e}}{L B \epsilon /(n-1)} \leq \frac{O P T_{c}}{L B \epsilon /(n-1)} \leq 2\left(\frac{n-1}{\epsilon}\right)
$$

we get that Step 3 of Algorithm FPAS-DCST definitely outputs a spanning tree. Let Heu be the tree output. Then we have that

$H e u_{c}=\sum_{e \in H e u_{c}} c_{e} \leq L B \epsilon /(n-1) \sum_{e \in H e u_{c}} \frac{c_{e}}{L B \epsilon /(n-1)} \leq L B \epsilon /(n-1)\left(\sum_{e \in H e u_{c}}\left\lfloor\frac{c_{e}}{L B \epsilon /(n-1)}\right\rfloor+1\right)$.

But since Step 3 of ALgORITHM FPAS-DCST outputs the spanning tree with minimum $c$-cost we have that

$$
\sum_{e \in H e u_{c}}\left\lfloor\frac{c_{e}}{L B \epsilon /(n-1)}\right\rfloor \leq \sum_{e \in O P T}\left\lfloor\frac{c_{e}}{L B \epsilon /(n-1)}\right\rfloor
$$

Therefore

$$
H e u_{c} \leq L B \epsilon /(n-1) \sum_{e \in O P T}\left\lfloor\frac{c_{e}}{L B \epsilon /(n-1)}\right\rfloor+\epsilon L B \leq \sum_{e \in O P T} c_{e}+\epsilon O P T_{c} \leq(1+\epsilon) O P T_{c} .
$$

This proves the claim. 
Lemma 8.6 The running time of ALGORITHM FPAS-DCST is $O\left(\frac{n}{\epsilon} p\left(n, \frac{n}{\epsilon}\right) \log C_{h i}\right)$.

Proof: From Claim 8.4 we see that Step 2 2 of ALgORITHM FPAS-DCST takes time $O\left(\frac{n}{\epsilon} p\left(n, \frac{n}{\epsilon}\right) \log C_{h i}\right)$ while Step 3 takes time $O\left(\frac{2 n}{\epsilon} p\left(n, \frac{2 n}{\epsilon}\right)\right)$. Hence the running time of AlgORITHM FPAS-DCST is $O\left(\frac{n}{\epsilon} p\left(n, \frac{n}{\epsilon}\right) \log C_{h i}\right)$.

Lemmas 8.6 and 8.5 yield:

Theorem 8.7 For the class of treewidth-bounded graphs, there is an FPAS for the (Diameter, Total cost, Spanning tree)-bicriteria problem with performance guarantee $(1,1+\epsilon)$.

As mentioned before, similar theorems hold for the other problems in Table 2 and all these results extend directly to Steiner trees.

\subsection{Near-Optimal Broadcast Schemes}

The polynomial-time algorithm for the (Degree, Diameter, Spanning tree)-bicriteria problem for treewidth-bounded graphs can be used in conjunction with the ideas presented in [Ra94] to obtain near-optimal broadcast schemes for the class of treewidth-bounded graphs. As mentioned earlier, these results generalize and improve the results of Kortsarz and Peleg [KP92].

Given an unweighted graph $G$ and a root $r$, a broadcast scheme is a method for communicating a message from $r$ to all the nodes of $G$. We consider a telephone model in which the messages are transmitted synchronously and at each time step, any node can either transmit or receive a message from at most one of its neighbors. The minimum broadcast time problem is to compute a scheme that completes in the minimum number of time steps. Let $O P T_{r}(G)$ denote the minimum broadcast time from root $r$ and let $O P T(G)=\operatorname{Max}_{r \in G} O P T_{r}(G)$ denote the minimum broadcast time for the graph from any root. The problem of computing $O P T_{r}(G)$ - the minimum rooted broadcast time problem - and that of computing $O P T(G)$ - the minimum broadcast time problem are both NP-complete for general graphs [GJ79]. It is easy to see that any approximation algorithm for the minimum rooted broadcast time problem automatically yields an approximation algorithm for the minimum broadcast time problem with the same performance guarantee. We refer the readers to Ra94 for more details on this problem. Combining our approximation algorithm for (Diameter, Total cost, Spanning tree)bicriteria problem with performance guarantee $(1,1+\epsilon)$ for the class of treewidth bounded graphs with the observations in Ra94 yields the following theorem.

Theorem 8.8 For any class of treewidth-bounded graphs there is a polynomial-time $O\left(\frac{\log n}{\log \log n}\right)$ approximation algorithm for the minimum rooted broadcast time problem and the minimum broadcast time problem. 


\section{Concluding Remarks}

We have obtained the first polynomial-time approximation algorithms for a large class of bicriteria network design problems. The objective function we considered were (i) degree, (ii) diameter and (iii) total cost. The connectivity requirements considered were spanning trees, Steiner trees and (in several cases) generalized Steiner trees. Our results were based on the following three ideas:

1. A binary search method to convert an $(\alpha, \beta)$-approximation algorithm for $(\mathbf{A}, \mathbf{B}, \mathbf{S})$-bicriteria problems to a $(\beta, \alpha)$-approximation algorithm for $(\mathbf{B}, \mathbf{A}, \mathbf{S})$-bicriteria problems.

2. A parametric search technique to devise approximation algorithms for $(\mathbf{A}, \mathbf{A}, \mathbf{S})$-bicriteria problems. We note that Theorem 6.3 is very general. Given any $\rho$-approximation algorithm for minimizing the objective $\mathbf{A}$ in the subgraph-class $\mathbf{S}$, Theorem 6.3 allows us to produce a $(2 \rho, 2 \rho)$ approximation algorithm for the (A, A, S)-bicriteria problem.

3. A cluster based approach for devising approximation algorithms for certain categories of $(\mathbf{A}, \mathbf{B}, \mathbf{S})$ bicriteria problems.

We also devised pseudopolynomial time algorithms and fully polynomial time approximation schemes for a number of bicriteria network design problems for the class of treewidth-bounded graphs.

\section{Subsequent work}

During the time when this paper was under review, important progress has been made in improving some of the results in this paper. Recently, Ravi and Goemans [RG95] have devised a $(1,1+\epsilon)$ approximation scheme for the (Total Cost, Total Cost, Spanning tree) problem. Their approach does not seem to extend to devising approximation algorithms for more general subgraphs considered here. In [KP97], Kortsarz and Peleg consider the (Diameter, Total Cost, Steiner tree) problem. They provide polynomial time approximation algorithms for this problem with performance guarantees $(2, O(\log n))$ for constant diameter bound $D$ and $\left(2+2 \epsilon, n^{\epsilon}\right)$ for any fixed $0<\epsilon<1$ for general diameter bounds. Improving the performance guarantees for one or more of the problems considered here remains an interesting direction for future research.

Acknowledgements: We would like to thank an anonymous referee for several useful comments and suggestions. We thank Sven Krumke (University of Würzberg) for reading the paper carefully and providing several useful comments. In particular, both pointed an error in the original proof of Theorem 5.3. We thank Professors S. Arnborg and H. L. Bodlaender for pointing out to us the equivalence between treewidth bounded graphs and decomposable graphs. We thank A. Ramesh for bringing [KP+93] to our attention. We also thank Dr. V. Kompella for making his other papers available to us. Finally, we thank the referees of ICALP' 95 for their constructive comments and suggestions. 


\section{References}

[AS97] S. Arora and M. Sudan, "Improved low-degree testing and its applications," Proc. 29th Annual ACM Symposium on Theory of Computing (STOC), 485-496 (1997).

$[\mathrm{AB}+90]$ B. Awerbuch, A. Baratz, and D. Peleg, "Cost-sensitive analysis of communication protocols," Proceedings of the 9th Symposium on Principles of Distributed Computing (PODC), pp. 177-187 (1990).

[AC+93] S. Arnborg, B. Courcelle, A. Proskurowski and D. Seese, "An Algebraic Theory of Graph Reductions," Journal of the ACM (JACM), vol. 40:5, pp. 1134-1164 (1993).

[AK+95] A. Agrawal, P. Klein and R. Ravi, "When trees collide: an approximation algorithm for the generalized Steiner problem on networks," SIAM Journal on Computing, vol.24, pp. 440-456 (1995).

[AL+91] S. Arnborg, J. Lagergren and D. Seese, "Easy Problems for Tree-Decomposable Graphs," Journal of Algorithms, vol. 12, pp. 308-340 (1991).

[Bo88] H.L. Bodlaender, "Dynamic programming on graphs of bounded treewidth," Proceedings of the 15th International Colloquium on Automata Language and Programming, LNCS vol. 317, pp. 105-118 (1988).

[BK90] A. Bookstein and S.T. Klein, "Construction of Optimal Graphs for Bit-Vector Compression," Proc. 13th ACM-SIGIR, vol. 16, pp. 387-400 (1990).

[BL+87] M.W. Bern, E.L. Lawler and A.L. Wong, "Linear -Time Computation of Optimal Subgraphs of Decomposable Graphs," Journal of Algorithms, vol. 8, pp. 216-235 (1987).

[CG82] P. M. Camerini, and G. Galbiati, “The bounded path problem,” SIAM Journal on Algebraic and Discrete Methods vol. 3, no. 4, pp. 474-484 (1982).

[Ch77] R. Chandrasekaran, "Minimum Ratio Spanning Trees," Networks, vol. 7, pp. 335-342, (1977).

[CLR] T.H. Cormen, C.E. Leiserson, and R.L. Rivest, Introduction to Algorithms, McGraw-Hill Book Co., 1990.

[Ch91] C.-H. Chow, "On multicast path finding algorithms," Proceedings of IEEE INFOCOM 1991, pp. 1274-1283 (1991).

[CK95] P. Crescenzi and V. Kann, "A compendium of NP optimization problems," Manuscript, (1995).

[FW+85] A. Frank, L. Wittie, and A. Bernstein, "Multicast communication in network computers," IEEE Software, vol. 2, no. 3, pp. 49-61 (1985).

[GG+95] J. L. Ganley, M. J. Golin and J. S. Salowe, "The multi-weighted spanning tree problem," Proceedings of the First Conference on Combinatorics and Computing (COCOON), Springer Verlag, LNCS pp. 141-150 (1995).

[GJ79] M. R. Garey and D. S. Johnson, Computers and intractability: A guide to the theory of NP-completeness, W. H. Freeman, San Francisco (1979).

[GW95] M. X. Goemans and D. P. Williamson, "A general approximation technique for constrained forest problems," SIAM Journal on Computing, Vol. 24, 1995, pp. 296-317. 
[Ha92] R. Hassin, "Approximation schemes for the restricted shortest path problem," Mathematics of Operations Research, vol. 17, no. 1, pp. 36-42 (1992).

[HL+89] J. Ho, D.T. Lee, C.H. Chang and C.K. Wong, "Bounded diameter spanning tree and related problems," Proceedings of the Annual ACM Symposium on Computational Geometry, pp. 276-282 (1989).

[Ho95] D. Hochbaum, Approximation algorithms for NP-hard problems, D.S. Hochbaum Ed., PWS Publishing Company, Boston, MA (1995).

[KJ83] B. Kadaba and J. Jaffe, "Routing to multiple destinations in computer networks," IEEE Transactions on Communications, Vol. COM-31, pp. 343-351 (March 1983).

[KR+93] S. Khuller, B. Raghavachari, and N. Young, "Balancing minimum spanning and shortest path trees," Algorithmica, vol. 14 (4), pp. 305-321, (1995).

[KP+92A] V.P. Kompella, J.C. Pasquale and G.C. Polyzos, "Multicasting for multimedia applications," Proceedings of IEEE INFOCOM 1992 (May 1992).

[KP+93] V.P. Kompella, J.C. Pasquale and G.C. Polyzos, "Multicast routing for multimedia communication," IEEE/ACM Transactions on Networking, pp. 286-292 (1993).

[KP92] G. Kortsarz and D. Peleg, "Approximation algorithms for minimum time broadcast," SIAM Journal on Discrete Mathematics, Vol. 8, No. 3, pp. 401-427 1995.

[KP97] G. Kortsarz and D. Peleg, "Approximating Shallow Light Trees," Proceedings of the Eighth Annual ACM-SIAM Symposium on Discrete Algorithms, pp. 103-110 (1997).

[Me83] N. Megiddo, "Applying parallel computation algorithms in the design of serial algorithms," Journal of the ACM (JACM), vol. 30, pp. 852-865, (1983).

[Ph+93] C. Phillips, "The Network Inhibition Problem," Proceedings of the 25th Annual ACM Symposium on the Theory of Computing, pp. 776-785, (1993).

[Ra94] R. Ravi, "Rapid rumor ramification: approximating the minimum broadcast time," Proceedings of the 35th Annual IEEE Foundations of Computer Science, pp. 202-213 (1994).

[RG95] R. Ravi and M. Goemans, "The constrained spanning tree problem," to appear in the Proceedings of the 5th Scandinavian Workshop on Algorithmic Theory, 1996.

[RM+93] R. Ravi, M. V. Marathe, S. S. Ravi, D. J. Rosenkrantz, and H.B. Hunt III, "Many birds with one stone: multi-objective approximation algorithms," Proceedings of the 25th Annual ACM Symposium on the Theory of Computing, pp. 438-447 (1993). (Expanded version appears as Brown University Technical Report TR-CS-92-58.)

[RS97] R. Raz and S. Safra, "A sub-constant error-probability low-degree test, and a sub-constant error-probability PCP characterization of NP," Proc. 29th Annual ACM Symposium on Theory of Computing, 475-484 (1997).

[Wa87] A. Warburton, "Approximation of Pareto optima in multiple-objective, shortest path problems," Operations Research, vol. 35, pp. 70-79 (1987).

[ZP+94] Q. Zhu, M. Parsa, and W.W.M. Dai, "An iterative approach for delay-bounded minimum Steiner tree construction," Technical Report UCSC-CRL-94-39, UC Santa Cruz (1994). 


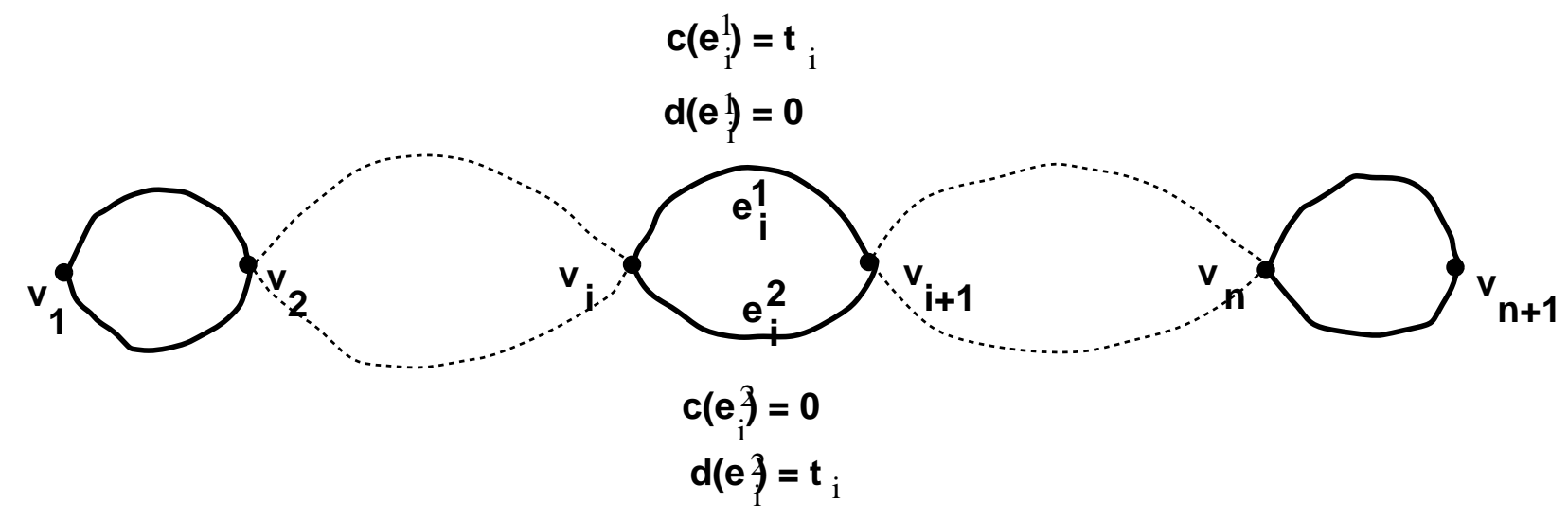

